\title{
Platform and State Estimation Design of a Small-Scale UAV Helicopter System
}

\author{
Shuai Tang, Xingju Lu, and Zhiqiang Zheng \\ College of Mechatronic Engineering and Automation, National University of Defense Technology, Changsha 410073, China \\ Correspondence should be addressed to Shuai Tang; tangshuai@nudt.edu.cn
}

Received 24 June 2013; Revised 17 October 2013; Accepted 17 October 2013

Academic Editor: Hui Hu

Copyright (C) 2013 Shuai Tang et al. This is an open access article distributed under the Creative Commons Attribution License, which permits unrestricted use, distribution, and reproduction in any medium, provided the original work is properly cited.

\begin{abstract}
This paper presents the development of a small-scale unmanned aerial vehicle (UAV) helicopter system based on a Raptor 90 hobby helicopter. Firstly, onboard avionics system and ground station are designed carefully. The onboard avionics system mainly consists of sensors, flight control board, RF modem, and power supply system. The ground station comprises a computer and a RF modem. The main function of ground station is to monitor the status of onboard avionics. To avoid the effect of noises, some efficient sensor data processing and integrated navigation algorithms are designed and implemented on the onboard avionics. As a result, the constructed system exhibits low weight, small size, antivibration, and low power consumption; the essential information for system identification and automatic control can be easily acquired. Several ground and flight tests have been performed to verify the feasibility and reliability of the total system. The results show that it is sufficient for system identification and automatic control.
\end{abstract}

\section{Introduction}

There has been considerable interest in research of unmanned aerial vehicle (UAV) in both academic and industrial areas recently. Researchers have explored the considerable potential of UAVs in various military and civil applications [14], such as border patrol, reconnaissance, intelligent traffic monitoring, search and rescue, fire detection, to name only a few of the application domains. Among numerous kinds of UAVs, small-scale helicopter has attracted much attention and effort of researchers due to its unique characteristics. For example, it can take off and land vertically and perform flight ranging from hovering to cruising with agility and maneuverability. As an underactuated, dynamically fast, and unstable system, it is considered to be an ideal platform for nonlinear control law research. Meanwhile, it is also an excellent test bed for path planning algorithms [5]. Actually, as a complex electromechanical system, helicopter itself is also a challenging object of research and much research can be conducted, such as modeling and system identification, autonomous taking off and landing control. In addition, equipped with different payload sensors, it can complete different missions. However, developing a helicopter platform capable of conducting those researches is the first step for research.

Designing and implementing a small-scale UAV helicopter system is a challenging job. Difficulty may come from various aspects such as selection of platform components, design of avionics and software, vibration isolation, design of sensor data processing, and integration algorithm. With the growth of electronic technology, onboard avionics are shrinking in size and weight. So, it is possible to develop onboard avionics for a small-scale UAV helicopter with attributes of low-cost, small size, light weight, and high performance. A number of research institutes have paid much effort. For example, M.I.T [6, 7], Carnegie Mellon University [8-10], Georgia Technology Institute [11-13], and so on have developed their own autonomous helicopter in the past decade. Recently, the UAV team in the National University of Singapore has carried out many researches on designing hardware $[14,15]$ and software $[16,17]$ of UAV helicopter. In addition, some other institutes have also constructed their own UAV helicopter platforms [18-21]. Generally, most UAV helicopters are constructed based on commercial hobby helicopter models due to their low cost. 
The goal of our group is to develop a low-cost smallscale UAV helicopter which can take off, cruise, and land autonomously. Meanwhile, it also can be treated as a test platform for control design. This objective places many requirements on the design work. The avionics should be able to collect the control inputs and all states of the helicopter, for identifying the mathematical model. It also should have strong information processing capability to perform accurate state estimation and high bandwidth feedback control. Another requirement is safety. The small-scale UAV helicopter, also called aerial robot, is quite different from those robots working on the ground, which are slower, simpler, and safer compared with the former. It needs higher reliability on the avionics and software. Once out of control in the air, it is dangerous for researchers and instruments. Then the manual control channel should be retained so that the control authority can be switched between human pilot and onboard avionics. Vibration isolation is also very important. The main rotor and engine, which are the main vibration sources in helicopter system, introduce heavily noise into the onboard sensors. More seriously, such strong vibration may destroy the onboard avionics system. Then, vibration isolator is necessary to weaken such effect.

In addition to the aforementioned, data processing algorithms are indispensable for state estimation and data fusion, which are very important but rarely mentioned in the available literature. Because some states such as velocity and position measured directly cannot satisfy the requirements and some signals are polluted by noises. How to obtain accurate navigation signals is a challenging problem for UAV helicopter. Unlike fix-wing UAV, the navigation of UAV helicopter cannot be done only using a single antenna Global Position System (GPS) receiver [22], because the dynamics of UAV helicopter is much faster. It is considered that navigation system requires data fusion. Those algorithms must be designed based on the processing capability of onboard processor. Commonly, the capability of the onboard system is limited by payload and power consumption severely. Then, some efficient but simplified algorithms are desired.

The outline of this paper is as follows. In Section 2, we introduce the framework of the UAV helicopter; the main attention is focused on the components selection and hardware system design. In Section 3, the design of inertial sensor data processing algorithm is illustrated. The GPS/INS integrated navigation algorithm and altitude estimation algorithm are designed in Section 4. Some ground and flight tests are performed in Section 5, and the feasibility and reliability of the system is evaluated. Finally, the conclusions and future work are presented in Section 6.

\section{Framework of Helicopter Platform}

The UAV helicopter platform we have eventually constructed is shown in Figure 1, which consists of the following four parts: (1) a Remote Control (RC) hobby helicopter, (2) a suit of onboard avionics system, (3) a ground station, and (4) a RC transmitter. The hobby helicopter is the base of the physical platform to be upgraded by the rest of the system.

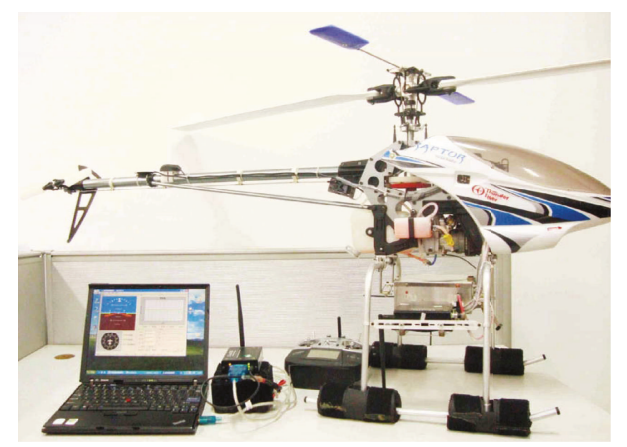

FIGURE 1: The overview of UAV helicopter system.

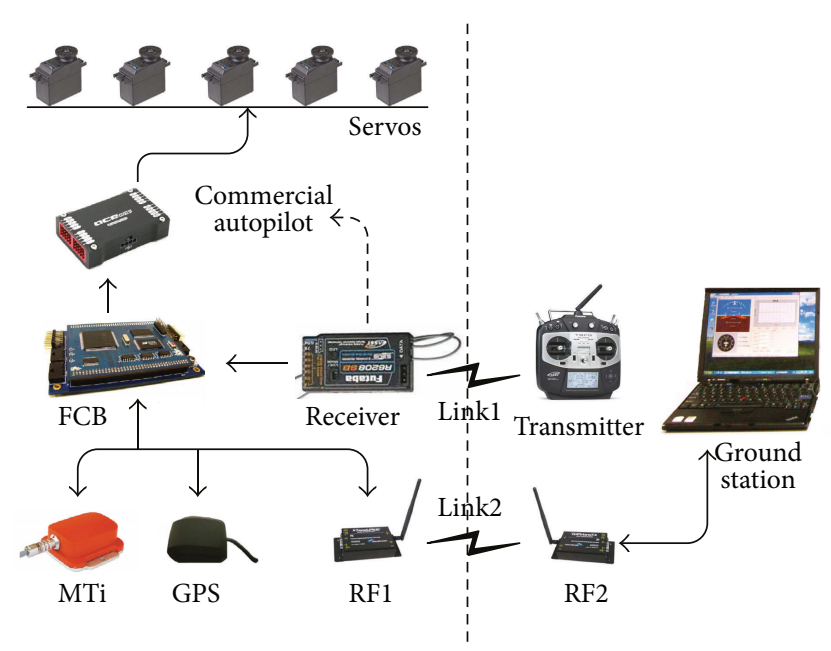

Left: onboard system

Right: ground station

FIGURE 2: The framework of UAV helicopter system.

The onboard avionics play an important role which are responsible for receiving the command from ground station and RC transmitter, collecting necessary data such as the attitude, angular rate, acceleration, velocity, the status of the power supply system, driving the servo actuators to control the helicopter, logging data to SD card, and transmitting some essential data to ground station. The ground station is used to monitor the status of the UAV helicopter through communicating with the onboard avionics. The RC transmitter is treated as a manual control system and the pilot can switch to manual control at any time. For a small-scale helicopter, an important index is that the cost should be minimum. In this section, the framework of the UAV helicopter platform is illustrated in detail. Figure 2 shows an overview of the UAV helicopter system. Much attention has been paid to select the hardware components and design the onboard avionics and ground station. To minimize the total cost, only some lowcost components are considered.

2.1. RC Hobby Helicopter. The basic rotorcraft we have selected is a high quality RC bare helicopter, Raptor 90, which 


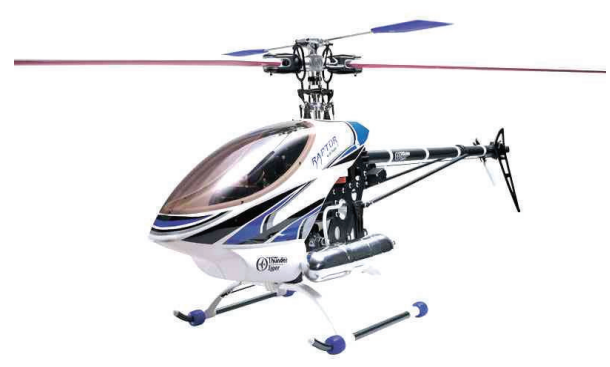

FIGURE 3: Raptor 90 hobby helicopter.

TABLE 1: Specifications of raptor 90.

\begin{tabular}{lc}
\hline Specification & Raptor 90 \\
\hline Length of fuselage & $1410 \mathrm{~mm}$ \\
Width of fuselage & $190 \mathrm{~mm}$ \\
Main rotor diameter & $1605 \mathrm{~mm}$ \\
Tail rotor diameter & $260 \mathrm{~mm}$ \\
Gear ratio (engine : main : tail) & $8.27: 1: 4.65$ \\
Full equipped weight & $4.8 \mathrm{~kg}$ \\
\hline
\end{tabular}

is designed for F-3D acrobatic flight, as shown in Figure 3. It has two-blade teetering rotor augmented with Bell-Hiller stabilizing bar. The stabilizing bar acts as a damper to reduce control sensitivity to facilitate manual control. Some key physical parameters of it are listed in Table 1. Five digital servo actuators are instrumented to drive the helicopter. All servo actuators we have selected are digital type for the sake of rapid response. In detail, three BLS253 brushless motors manufactured by Futaba are selected as aileron, elevator, and collective pitch actuator to tilt the swash plate to generate the roll, pitch moments and change the magnitude of main rotor thrust cooperating with the throttle servo actuator; a Futaba S9254 high-speed servo actuator is used to change the collective pitch angle of the tail rotor; a DS1210 digital servo is selected to control the throttle of the engine. Actually, the rotation of main rotor is governed at a invariable speed, and the pitch angle of main rotor is controlled to change the magnitude of thrust. The engine is a vital component in helicopter system. In order to get large payload, an OS-91HZ$\mathrm{R}$ alcohol-powered engine is equipped to supply power for the main and tail rotor. It can output about $3.6 \mathrm{ps}$ at $15500 \mathrm{rpm}$. Then, the gross take off weight can reach to $12 \mathrm{~kg}$. With $480 \mathrm{cc}$ fuel, each flight can last 12 minutes.

It is not easy to control a hobby helicopter, especially for beginners. A skilled pilot is needed to carry out research works. One needs much training time to improve the operation. To make the bare hobby helicopter easy to be operated, a low-cost commercial autopilot with modest performance is equipped as stability augmentation system, which is helpful at the beginning to avoide an accident. And it is retained in our subsequent research. However, there is no communication interface for development and we cannot get any signal from it. Actually, in our design, the autopilot is just used as engine

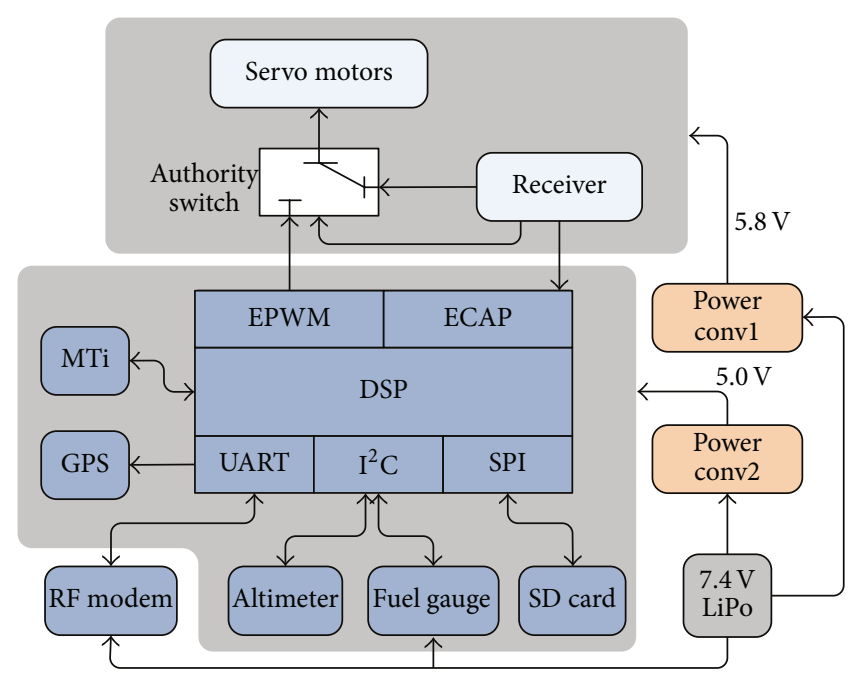

FIGURE 4: Basic principle of the onboard avionics system.

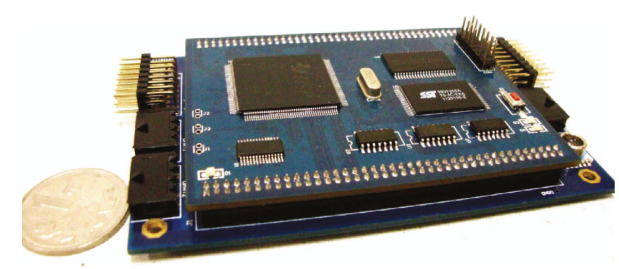

FIGURE 5: Flight control board in our lab.

governor, angular vector control system (yaw rate gyro) for yaw channel, and servo driver.

2.2. Onboard Avionics System. The onboard avionics system mainly consist of the flight control board, sensors, radio frequency (RF) modem, and power supply system. The basic design criterions are (1) to be able to provide relative strong processing capability to implement complicated algorithms and rich peripherals to connect with the sensors, (2) to be able to provide a full range of sensor data (3) to be small in size and light in weight. The working principle of the onboard avionics system is shown in Figure 4.

2.2.1. Flight Control Board. The main tasks of the flight control board include (1) collecting data from sensors and commands from RC receiver, (2) conducting sensor data processing algorithms, (3) implementing flight control law and control servo actuators through servo driver, (4) logging data into SD card, and (5) communicating with ground station through RF modem.

A small-size flight control board which satisfies all requirements has been developed in our lab, as is shown in Figure 5. The digital signal controller (DSC) TMS320F28335 is selected as CPU, the work frequency of which is up to $150 \mathrm{MHz}$ and the on-chip float-point unit processes rapid processing speed. It contains various peripherals, including 
3 Serial Communication Interface (SCI) modules, 1 Serial Peripheral Interface (SPI) module, 2 Controller Area Network (CAN) bus modules, 1 Interintegrated Circuit $\left(\mathrm{I}^{2} \mathrm{C}\right)$ bus module, 6 Enhanced Capture (ECap) modules, and 6 enhanced Pulse Width Modulator (PWM) modules. Then it is easy to connect with the selected sensors and other peripherals. On the board, 3 SCI modules are used to communicate with Attitude Heading Reference System (AHRS), GPS receiver, and $\mathrm{RF}$ modem, respectively; the $\mathrm{I}^{2} \mathrm{C}$ bus module is used to connect with altimeter and battery monitor, and the CAN bus modules are reserved for the future to expand payload sensors. The enhanced capture modules are used to capture the width of PWM signals from RC receiver. It is important for system identification to collect those signals. The enhanced PWM modules are used to output the control signals of the servo actuators. Data logging is another task of avionics, which is very important for system identification and off-line analysis. The on-chip memory of the DSC is not enough and a 4 GB SD card is installed on the printed circuit board interfacing to the DSC with SPI protocol.

For safety, the control authority should be able to be switched between pilot and onboard DSC freely. That means both the signals from RC receiver and DSC could be able to control the servo actuators. Several data multiplexers are installed on the board for this purpose. In manual control mode, the RC receiver outputs are selected to drive the corresponding servo actuators. On the other hand, when autonomous control mode is set, the control signals from DSC instead of from RC receiver are sent to servo driver, but the RC pilot can switch to manual control mode in any case of emergency with a switch on RC transmitter immediately. The DSC can sample the $\mathrm{RC}$ receiver outputs all the time. The default setting of the data multiplexers is to let the $\mathrm{RC}$ receiver signals go through, which guarantees that the pilot still can control the helicopter even when the DSC is powered off.

2.2.2. Avionic Sensors. The principles of sensor selection are the sensor packet that should be able to provide the essential signals for system identification and flight control and the measure range that should cover the entire flight envelope. Generally, the measuring range of $3 \mathrm{D}$ accelerations and $3 \mathrm{D}$ angular rates should be larger than $\pm 2 \mathrm{~g}, \pm 150^{\circ}$, respectively, for small-scale UAV helicopter [14]. There are several vital sensors equipped on the helicopter: an AHRS, a GPS receiver, and a barometer. AHRS provides the inertial measurement, GPS receiver provides navigation information, and barometer is used to measure altitude.

A compact gyroenhanced AHRS, namely, MTi, shown in Figure 6, is selected as a kernel unit to deliver inertial measurement for our helicopter. It is designed using Microelectro Mechanical System (MEMS) technology. Its internal low-power signal processor provides drift-free three-axis orientation as well as calibrated three-axis acceleration, threeaxis angular rate, and three-axis earth-magnetic field data. Some key parameters of it are listed in Table 2. It is clear that it satisfies the measurement requirements of the helicopter.

Velocity and position in navigation frame are essential for flight control. Of cause, one can calculate them through

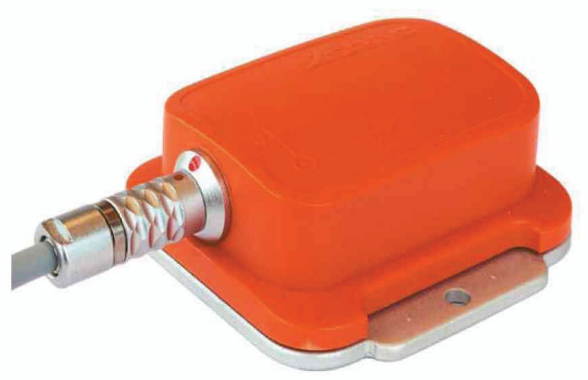

Figure 6: The appearance of MTi.

TABLE 2: Some parameters of MTi.

\begin{tabular}{lc}
\hline Specification & $\mathrm{MTi}$ \\
\hline Acceleration range & $\pm 50 \mathrm{~m} / \mathrm{s}^{2}$ \\
Angular rate range & $\pm 300 \mathrm{deg} / \mathrm{s}$ \\
Magnetometer range & $\pm 750 \mathrm{mGauss}$ \\
Roll/pitch accuracy & $<0.5 \mathrm{deg}$ \\
Heading accuracy & $<1.0 \mathrm{deg}$ \\
Output rate & User settable, $\max 120 \mathrm{~Hz}$ \\
Size & $58.7 \times 57.9 \times 23 \mathrm{~mm}$ \\
Weight & $50 \mathrm{~g}$ \\
\hline
\end{tabular}

integrating the acceleration cooperating with orientation, but the accuracy of the MEMS device is not reliable for a long time. In order to compensate for such disadvantage of $\mathrm{MTi}$, a low-cost GPS receiver based on u-blox module is installed in an antenna box and mounted on the tail boom, shown in Figure 7. Some key specifications of the receiver are listed in Table 3. It can output position (longitude, latitude, and altitude), velocity (magnitude, and direction) with $1 \mathrm{~Hz}$ update rate.

Due to the inaccuracy of GPS signals in altitude channel, the altitude signal from other sensor as reference for automatic taking off and landing is very important. Barometer is capable of providing altitude signal through detecting the change of air pressure. A high resolution barometer module, namely, MS5801, is adopted to measure air pressure. It is a new generation of high resolution altimeter sensor with an altitude resolution of $10 \mathrm{~cm}$. It can provide calibrated digital air pressure and temperature information. However, there are also some problems on altitude measurement using barometer alone, and the detail algorithm for altitude estimation will be illustrated in Section 4.

2.2.3. RF Modem. The wireless communication between onboard system and ground station is realized by a pair of Digi XTend-PKG RF modems. It is selected because of the compact appearance and high performance, that is, high throughput (up to $115.2 \mathrm{kbps}$ ), wide effective range (up to $64 \mathrm{~km}$ in the open field environment), and the working 


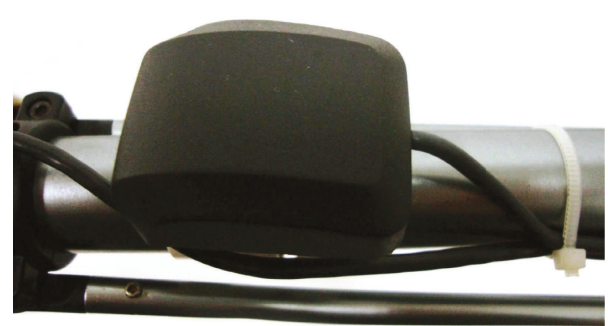

FIGURE 7: GPS module mounted on tail boom.

TABLE 3: Specifications of GPS module.

\begin{tabular}{lc}
\hline Specification & GPS module \\
\hline Model & NEO-6M \\
Sensitivity & $-161 \mathrm{dBm}$ \\
Update rate & $1 \mathrm{~Hz}$ \\
Position accuracy & $2.5 \mathrm{~m}$ \\
Time to first fix & $27 \mathrm{~s}($ Cold $) / 1 \mathrm{~s}$ (Hot) \\
\hline
\end{tabular}

frequency at $900 \mathrm{MHz}$ which is different from the working frequency of RC transmitter and receiver at $2.4 \mathrm{GHz}$. The RF power can be set through software configure ranging from $1 \mathrm{~mW}$ to $1 \mathrm{~W}$. The power supply voltage ranges from $7.0 \mathrm{~V}$ to $28 \mathrm{~V}$. The radio will produce electromagnetic interference, which may affect the rest of avionics, especially the flight control board and servo actuators. Then, the antenna needs to be put as far away as possible, as shown in Figure 1.

2.2.4. Power System. Based on the input voltage range and power consumption of each device on the UAV helicopter, a two-cell $7.4 \mathrm{~V}$ Lithium-Polymer (LiPo) battery pack with a capacity of $5600 \mathrm{mAh}$ is chosen to supply the total onboard system. The main consideration in the power supply design is the safety of the system. Even though there is just one battery pack, two power conversion modules are selected to maintain the whole system, as shown in Figure 4. One module with an output voltage of $5.0 \mathrm{~V} / 5 \mathrm{~A}$ is used to supply the flight control board and sensor packet. The other one, namely, REC$\mathrm{B} 6 \mathrm{~T}$, is equipped to output both $5.8 \mathrm{~V}$ for $\mathrm{RC}$ receiver and servo actuators and $1.5 \mathrm{~V}$ for engine glow plug ignition, which ensure that even when the power of DSC is out of order, the manual control channel can still work independently. Considering the high switching frequency of switch voltage regulator may cause electromagnetic interference, the two modules we have selected are both linear voltage regulators. The RF modem is powered by the battery pack directly. In addition to the two power conversion modules design, an ultracompact fuel-gauge system, MAX17041, is installed on the flight control board to monitor the status of the LiPo battery pack, which can output the real-time voltage and state of charge. It is easy to get the information at ground station when the helicopter is in flight.

2.3. Ground Station. The ground station is designed for human-UAV helicopter interaction. System configures and

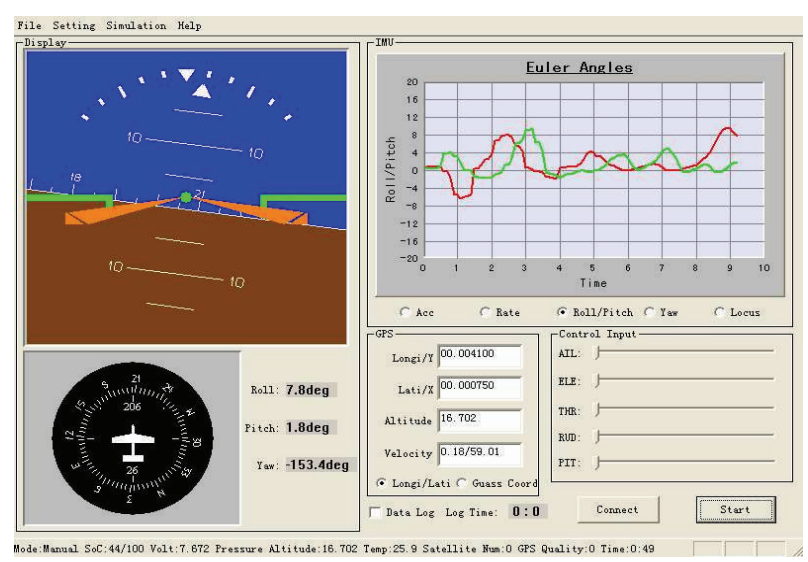

FIGURE 8: User interface of ground station.

task commands are generated at ground station and sent to the UAV helicopter, and flight data are transmitted to the ground station. A pair of RF modems depicted in Section 2.2.3 is used to connect the UAV helicopter with the ground station. A normal PC provides a friendly and easy-to-use graphical user interface for command generation and data view, as shown in Figure 8. The attitude of UAV helicopter is shown in artificial horizon and compass. The position of the helicopter can be displayed in GPS data area. Both GPS coordinate and Gauss coordinate can be selected. The graphical window on the right can be selected to display accelerations, angular rates, Euler angles, and motion trajectory of the helicopter in curve style.

In addition to the aforementioned data view function, the following two functions are realized on the ground station: reading the logged data stored in SD card and playing the role of a simulation terminal. Actually, high frequency real-time data transmission to ground station is not possible, which is limited by the bandwidth of the wireless communication. Then, the states of helicopter for system identification are recorded to SD card with a $100 \mathrm{~Hz}$ frequency in flight. After the flight has been completed, ground station is used to read the recorded data and save them in .txt format. When ground station plays the role of a simulation terminal, it is used to playback the recorded data or connect with MATLAB through UDP to validate control law.

2.4. Vibration Isolation. The vibrations on the UAV helicopter are mainly caused by the main rotor, the tail rotor, and the engine. The main rotor rotation is governed at $1650 \mathrm{rpm}$, correspondingly, the frequency of which is $27.5 \mathrm{~Hz}$. Due to the gear ratio of the driving system, the vibration frequencies of tail rotor and engine are $50 \mathrm{~Hz}$ and $227.5 \mathrm{~Hz}$, respectively. Such harmful vibrations will introduce noises and errors and impose danger to the onboard system. Then it is essential to build a proper type of antivibration subsystem to protect the onboard system. In this research, four wire-rope isolators are selected to install the avionics box to attenuate the vibration, as shown in Figure 9. All the avionic components except RF modem are installed in a $15 \times 13 \times 6 \mathrm{~cm}$ stainless steel box, 


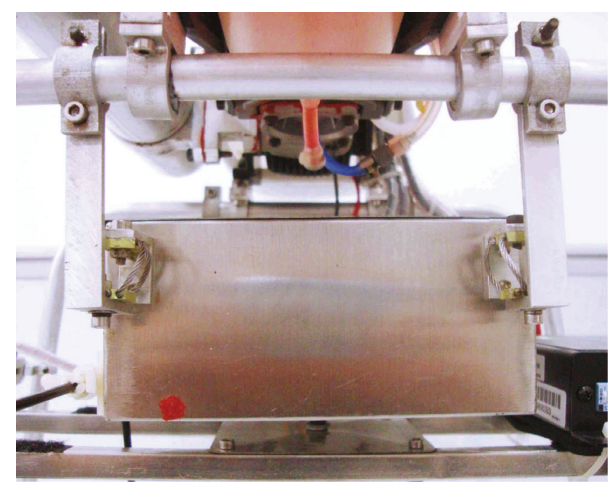

FIgURE 9: Antivibration subsystem.

which is mounted onto the landing skid with the four wirerope isolators. Due to the natural frequency of $11 \mathrm{~Hz}$ and damping ratio of 0.012 , the isolators are capable of attenuating high frequency vibration inputs from the main frame to much lower levels. However, this is insufficient to deal with the vibrations and some software algorithms will be introduced in Section 3.

\section{MTi Data Processing}

The MTi is configured to output data with a $100 \mathrm{~Hz}$ frequency that consists of acceleration, angular rate, magnetic field data, and orientation. The orientation is set in Euler angles (roll, pitch, and yaw) mode, which is calculated by the MTi's proprietary sensor fusion algorithm. The design of the algorithm can be explained as a sensor fusion algorithm, where the measurement of gravity (measured by accelerometers) and magnetic north (measured by magnetometers) are used to compensate for otherwise unlimited increasing (drift) errors from the integration of angular rates. Then, the orientation data can be used directly in our system.

3.1. Noise Analysis. However, the acceleration and angular rate are just unprocessed physical calibrated data. There is no additional filtering or other temporal processing applied to those signals. But those items are important for system identification and flight control; therefore, some additional data processing algorithms are necessary.

There are two types of noise for acceleration and angular rate, the MEMS sensor noise and the vibration of the helicopter. The MEMS sensor noise mainly consists of constant drift, time-varying drift, and observation noise. The vibration of the helicopter has been depicted in Section 2.4. In order to analyze the effect of those noises, two groups of data are sampled and the FFT of which are calculated, as shown in Figures 10 and 11. The angular rate along $z$-axis of body frame shown in Figure 10(a) was logged when the helicopter was on the ground and the engine was powered off. It is easy to conclude from the corresponding FFT result shown in Figure 10(b) that the sensor noise distributes over the entire frequency uniformly. Signal along the same axis logged during actual flight is shown in Figure 11. The amplitudes of
FFT in the vicinity of the $27.5 \mathrm{~Hz}$ are much larger than those of others, as shown in Figure 11(b), which are corresponding to the frequency of the main rotor rotation, $1650 \mathrm{rpm}$. It means that the vibration of the main rotor has coupled to the sensor data deeply. Actually, the effect of tail rotor and engine is much smaller.

3.2. Filter Design. In order to attenuate the effect of noises and improve the quality of sensor data, a two-stage cascade filter is proposed. The principle is shown in Figure 12. The first stage Kalman filter is used to deal with the MEMS sensor noise. A Chebyshev type low pass filter is designed as the second stage, which is responsible for the mentioned vibration. The error model of sensor is essential for Kalman filter design. Commonly, the measurement of MEMS gyroscope or accelerometer can be described as follows [23]:

$$
\begin{gathered}
x_{m}=x+b_{x 0}+b_{x}(t)+b_{x w}, \\
\dot{b}_{x}(t)=-\frac{1}{\tau} b_{x}(t)+w_{x 2},
\end{gathered}
$$

where, $x_{m}$ is the measurement, angular rate or acceleration; $x$ is the actual angular rate, or acceleration; $b_{x 0}$ is the constant drift; $b_{x}(t)$ is the time-varying drift, which often is modeled as first-order Markoff process (shown in (2)); $\tau$ is the time constant; $w_{x 2}$ is the drive noise, which is a stationary random process with zero expectation; and $b_{x w}$ is the observation noise. Then the total error dynamics can be described as the following three-order state space model:

$$
\left[\begin{array}{c}
\dot{x} \\
\dot{b}_{x} \\
\dot{b}_{x 0}
\end{array}\right]=\left[\begin{array}{ccc}
0 & 0 & 0 \\
0 & -\frac{1}{\tau} & 0 \\
0 & 0 & 0
\end{array}\right]\left[\begin{array}{c}
x \\
b_{x} \\
b_{x 0}
\end{array}\right]+\left[\begin{array}{ll}
1 & 0 \\
0 & 1 \\
0 & 0
\end{array}\right]\left[\begin{array}{l}
w_{x 1} \\
w_{x 2}
\end{array}\right] .
$$

Actually, it is difficult to model the derivative of angular rate or acceleration. So, the noise technique is adopted and the derivative is represented by a random noise $w_{x 1}$ with relative large variance [24].

The linear time-invariant model in (3) is suitable for standard Kalman filter implementation, and six independent Kalman filters are designed and implemented on the DSC for each state (3D angular rates and 3D accelerations), respectively.

The cutoff frequency of the low pass filter is an important parameter for the filter design in this system. Too low cutoff frequency may cause the avionics cannot capture some key dynamic characteristics we are interested in, but too high cutoff frequency will make against vibration isolation. Eventually, refer to [25], it is set at $10 \mathrm{~Hz}$ to let the rotorfuselage dynamics and the stabilizer dynamics pass through but isolate the vibration. At last, a five-order Chebyshev low pass filter is designed for each channel, similar to the first stage Kalman filter. The performance of the filters will be evaluated in Section 5.

\section{Integrated Navigation}

As is known to all, GPS is a common choice for positioning. However, GPS alone is incapable of providing continuous 


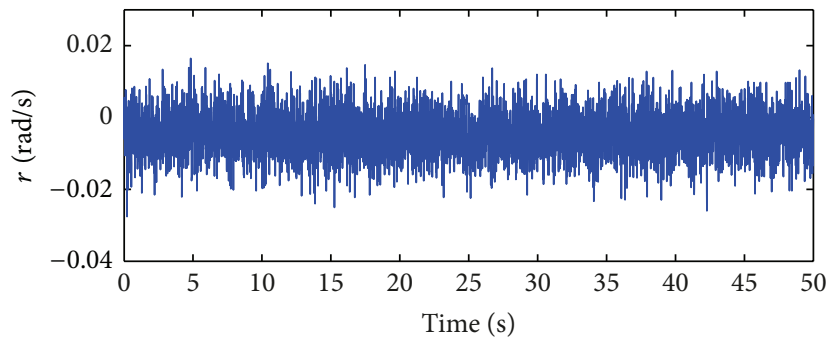

(a)

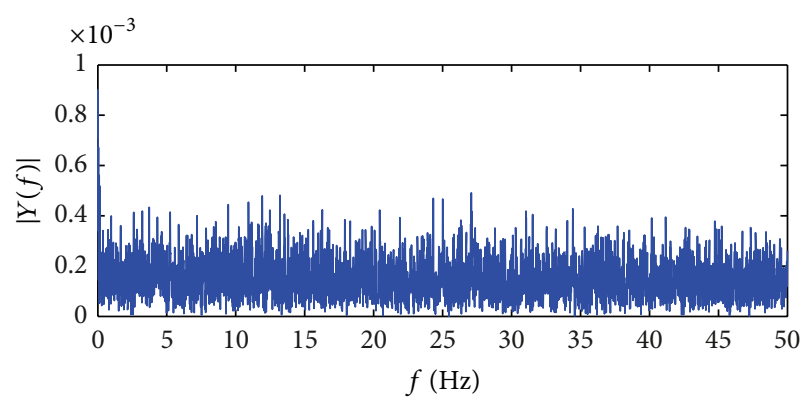

(b)

FIgURE 10: Noise analysis on the ground.

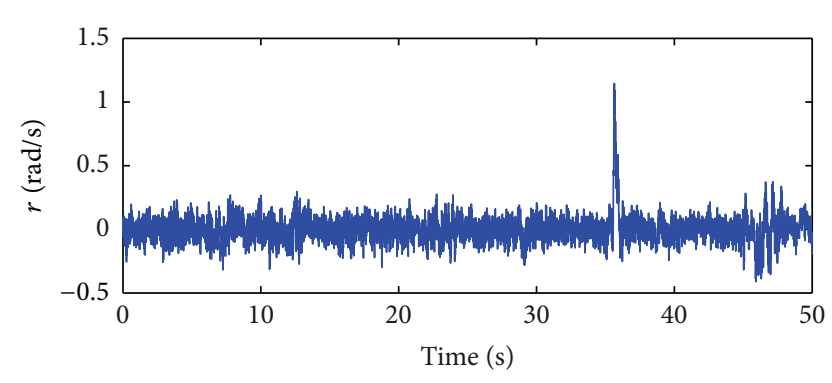

(a)

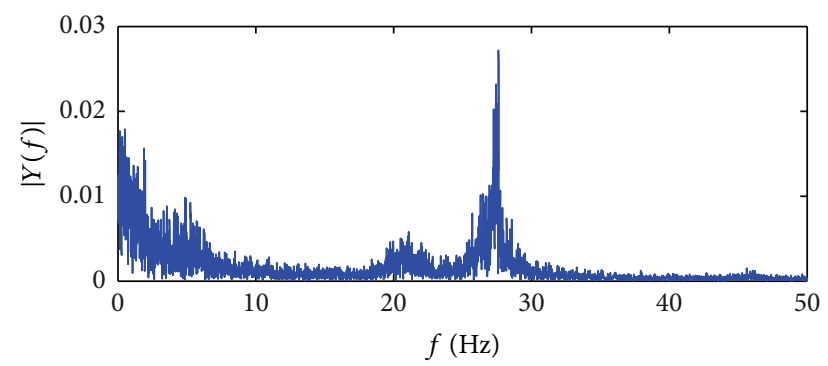

(b)

FIgURE 11: Noise analysis in flight.

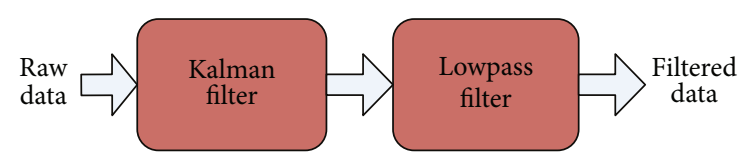

FIGURE 12: The principle of the two-stage filter.

and reliable positioning, because of its low update rate and inherent dependency on external electromagnetic signals. On the other hand, Inertial Navigation System (INS) has unbounded error growth since the error accumulates at each step. Therefore, in order to get believable velocity and position of UAV helicopter, integrated navigation is required, which is able to exploit individual strengths and offset the weakness of each individual subsystem.

In this section, the integrated navigation algorithm, integrating the information from MTi, GPS, and altimeter, used to estimate the velocity and position, is presented. It mainly consists of two submodules, and the main framework

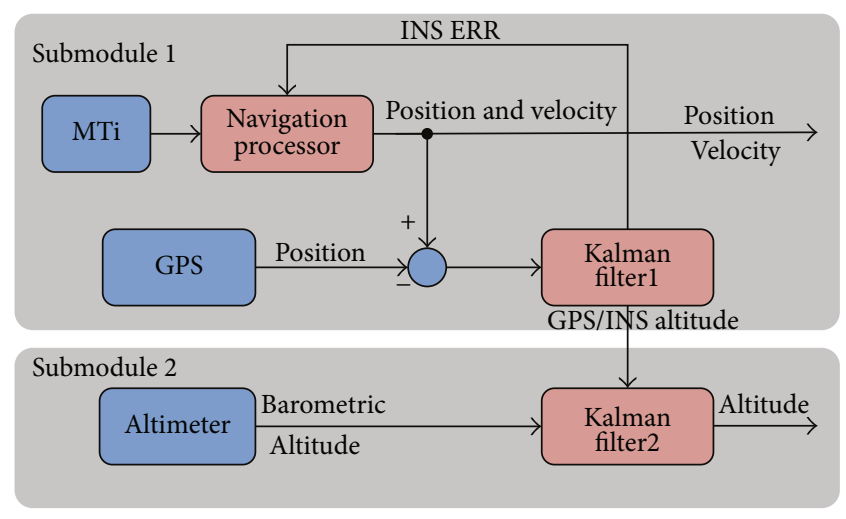

FIGURE 13: Framework of integrated navigation.

is shown in Figure 13. In the first submodule, GPS/INS integrated navigation algorithm is designed to estimate the velocity and position. However, the altitude from the GPS/INS integrated navigation is not reliable due to the inaccuracy of GPS in altitude channel. Then, considering the importance of altitude in flight control, the second submodule is designed to estimate the altitude independently, using the GPS aided altitude and the barometric altitude.

4.1. GPS/INS Integrated Navigation. Processed accelerations from the two-stage filters and orientation are used to calculate the inertial position and velocity. Actually, UAV helicopter always works in a small range and low speed spectrum. Then the effect of navigation frame rotation motion and variation of earths radius can be ignored. In addition, the accuracy of MTi is not enough to detect the earth rotation rate. Therefore, the terms related to the earth rotation rate in traditional INS mechanization can be neglected. North-East-Down (NED) coordinate system is adopted as the navigation frame in this paper, and then the inertial velocity update equation is simplified as

$$
\begin{gathered}
\dot{v}^{n}=C_{b}^{n} f^{b}+g^{n}, \\
v^{n}=\left[\begin{array}{lll}
v_{N} & v_{E} & v_{D}
\end{array}\right]^{T},
\end{gathered}
$$

where $C_{b}^{n}$ is the rotation matrix from body frame to navigation frame, which is calculated using the attitude angles 
directly instead of integrating the angular rate; $f^{b}$ is the output of accelerometer; and $g^{n}$ is the acceleration of gravity. Compared to the radius of earth, the altitude of UAV helicopter is always much smalled and can be ignored. Then inertial position update equations are simplified as

$$
\begin{gathered}
\dot{p}_{N}=v_{N}, \\
\dot{p}_{E}=\frac{v_{E}}{\cos L}, \\
\dot{h}=-v_{D},
\end{gathered}
$$

where $p_{N}$ and $p_{E}$ are the position of the small-scale helicopter along north and east, respectively; $L$ and $h$ represent the latitude, altitude, respectively.

The data fusion process of GPS/INS integrated navigation is done with a Kalman filter. And the errors of navigation states are selected as states of filter model, which makes sure the filter model is linear instead of nonlinear if navigation states are selected directly. The loosely coupled configuration is considered, which is easy to implement in engineering. In this configuration, both INS and GPS work independently, and the results from GPS are given as feedback to improve the performance of INS. The filter model is established based on INS, navigation signals obtained from GPS only are used as observation. In order to improve the processing speed, a simplified mathematic model is established, which will be discussed later. Meanwhile, the measurement we select is position error only. Because the velocity output of GPS is much later than the position, it is difficult to align the data from MTi and GPS if the position and velocity errors are both selected as measurement.

MTi is designed as an AHRS, the main function of which is to measure the orientation of the carrier. So, it is assumed that the output attitude is accurate enough and the error dynamic of which is not considered. It is assumed that the gravity is a constant. The error dynamics of MEMS sensors has been considered in Section 3.2, there is no need to repeat. At last, the velocity error update equation can be written as

$$
\delta \dot{v}^{n}=\left(C_{b}^{n} f^{b}\right) \times \psi+C_{b}^{n} \delta f^{b},
$$

where $\delta v^{n}$ is the velocity error vector; $\psi$ is the attitude error vector, treated as measurement noise; and $\delta f^{b}$ is the measurement error of accelerometer. And the statistic of $\psi$ and $\delta f^{b}$ can be found in product specification of MTi.

Considering that the position range of a small-scale helicopter is very small, it is reasonable to assume that the latitude is a constant. According to inertial position update equations, the position error update equations can be simplified as

$$
\begin{gathered}
\delta \dot{p}_{N}=\delta v_{N}, \\
\delta \dot{p}_{E}=\frac{\delta v_{E}}{\cos L}, \\
\delta \dot{h}=-\delta v_{D}
\end{gathered}
$$

where $\delta p_{N}, \delta p_{E}$, and $\delta h$ represent north position error, east position error, and altitude error, respectively.
At last, the error model for the integrated navigation Kalman filter can be concluded as follows:

$$
\begin{gathered}
\dot{\mathbf{X}}=\left[\begin{array}{c}
\mathbf{0}_{3 \times 6} \\
A_{1}
\end{array}\right] \mathbf{X}+\left[\begin{array}{c}
\left(C_{b}^{n} f_{b}\right)^{\times} \\
\mathbf{0}_{3 \times 3}
\end{array}\right] \psi+\left[\begin{array}{c}
C_{b}^{n} \\
\mathbf{0}_{3 \times 3}
\end{array}\right] \delta f^{b}, \\
\mathbf{X}^{T}=\left[\begin{array}{lllllll}
\delta v_{N} & \delta v_{E} & \delta v_{D} & \delta p_{N} & \delta p_{E} & \delta h
\end{array}\right]^{T}, \\
A_{1}=\left[\begin{array}{cccccc}
1 & 0 & 0 & 0 & 0 & 0 \\
0 & \frac{1}{\cos L} & 0 & 0 & 0 & 0 \\
0 & 0 & -1 & 0 & 0 & 0
\end{array}\right],
\end{gathered}
$$

where $(\cdot)^{\times}$represents the skew symmetric matrix of $(\cdot)$.

The measurement equation is obtained by comparing the GPS position to the INS position. The observation supplied to the Kalman filter is therefore expressed as follow:

$$
\mathbf{Z}=P_{\mathrm{INS}}-P_{\mathrm{GPS}}=\left[\begin{array}{c}
p_{\mathrm{EINS}}-L_{\mathrm{EGPS}} \\
p_{\mathrm{NINS}}-p_{\mathrm{NGPS}} \\
h_{\mathrm{INS}}-h_{\mathrm{GPS}}
\end{array}\right]
$$

Therefore, the observation $\mathbf{Z}$ related to the error state vector $\mathbf{X}$ can be written as follows:

$$
\mathbf{Z}=\left[\begin{array}{ll}
\mathbf{0}_{3 \times 3} & \mathbf{I}_{3 \times 3}
\end{array}\right] \mathbf{X}+\mathbf{v}
$$

where I represents identity matrix and $\mathbf{v}$ is the observation error.

Based on the extremely simplified model described in (8) and (11), the integrated navigation Kalman filter is designed and implemented on the DSC to estimate the navigation error, which is used to adjust the inertial navigation states (position and velocity). The filter is started after the GPS data has been valid so that the navigation states can be initialized easily by the first frame GPS data, and the performance of which will be evaluated in Section 5 .

4.2. Altitude Estimation. Barometer is used to measure altitude by detecting the change of air pressure. The air pressure can be converted to altitude with an empirical formula as follows:

$$
h_{\mathrm{bar}}=\frac{T_{b}}{\beta}\left[\left(\frac{p_{h}}{p_{b}}\right)^{-\beta R_{h} / g}-1\right] \text {, }
$$

where $T_{b}$ is the air temperature at sea level, $\beta$ is the temperature drop rate related to altitude, $p_{h}$ is the input air pressure, $p_{b}$ is the air pressure at sea level, and $R_{h}$ is the air constant. However, various factors may cause changes in air pressure, such as temperature and humidity. Meanwhile, $T_{b}, \beta$, and $p_{b}$ are also time varying, which will affect barometric altimeter measurement. As a result, the measurement of barometric altimeter cannot be used directly. In actual measurement, it is found that the barometric altitude for a fixed location in different time is quite different. But in one flight, the difference can be modeled as a constant bias, because of the short flight time. Another measurement is the GPS/INS 


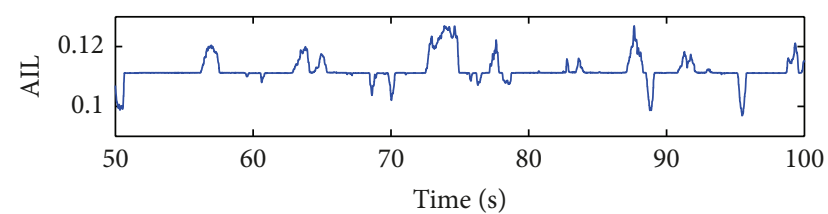

(a)

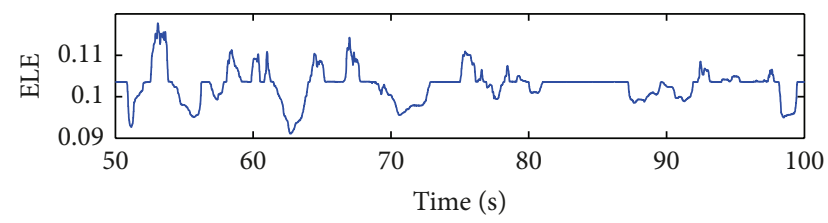

(b)

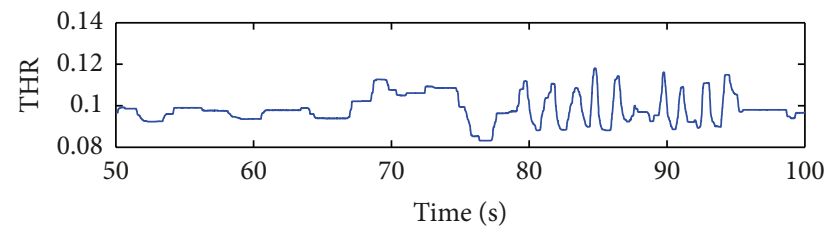

(c)

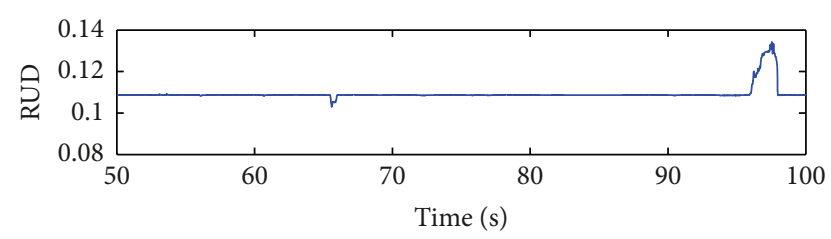

(d)

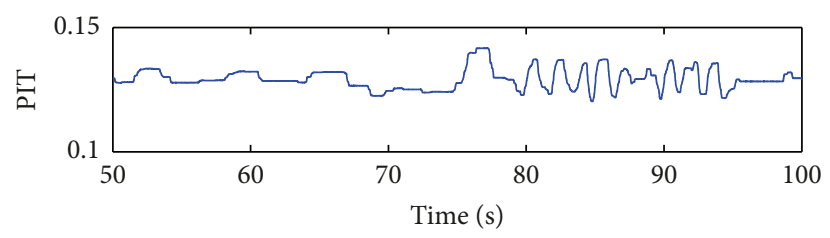

(e)

FIgure 14: Control inputs in flight test.

integrated navigation altitude. Then, the measurement model of the altitude can be expressed as follows:

$$
\begin{gathered}
h_{\mathrm{bar}}=h+b_{h}+\varepsilon_{1}, \\
h_{\mathrm{int}}=h+\varepsilon_{2},
\end{gathered}
$$

where $h_{\text {bar }}$ is the output of barometric altimeter and $h_{\text {int }}$ is the result of integrated navigation; $h$ is the true altitude; $b_{h}$ is the constant bias of barometric altimeter; and $\varepsilon_{1}$ and $\varepsilon_{2}$ are the measurement noises. Actually, $\varepsilon_{2}$ is much larger than $\varepsilon_{1}$.

Selecting the altitude $h$, the velocity $v_{h}$, and acceleration $a_{h}$ along altitude channel and the constant bias $b_{h}$ as state variables, the altitude dynamics are established as follow:

$$
\left[\begin{array}{c}
\dot{h}^{h} \\
\dot{v}_{h} \\
\dot{a}_{h} \\
\dot{b}_{h}
\end{array}\right]=\left[\begin{array}{llll}
0 & 1 & 0 & 0 \\
0 & 0 & 1 & 0 \\
0 & 0 & 0 & 0 \\
0 & 0 & 0 & 0
\end{array}\right]\left[\begin{array}{c}
h \\
v_{h} \\
a_{h} \\
b_{h}
\end{array}\right]+\left[\begin{array}{l}
0 \\
0 \\
1 \\
0
\end{array}\right] w .
$$

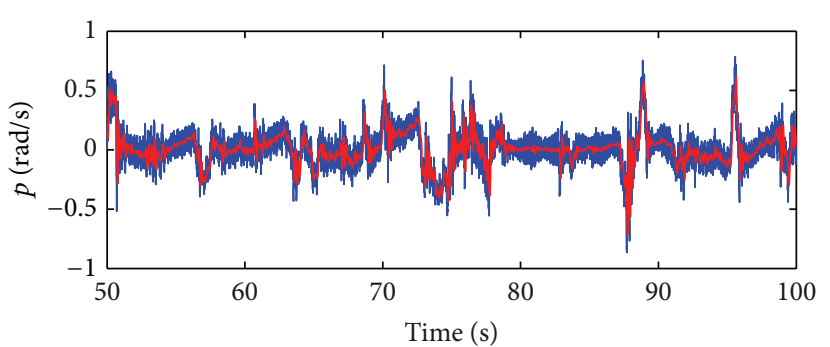

(a)

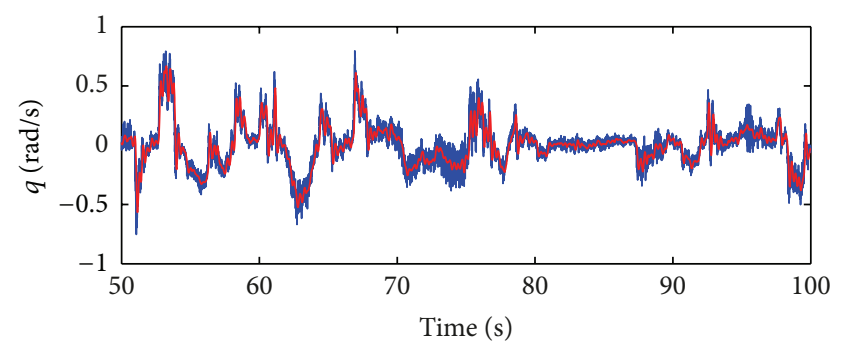

(b)

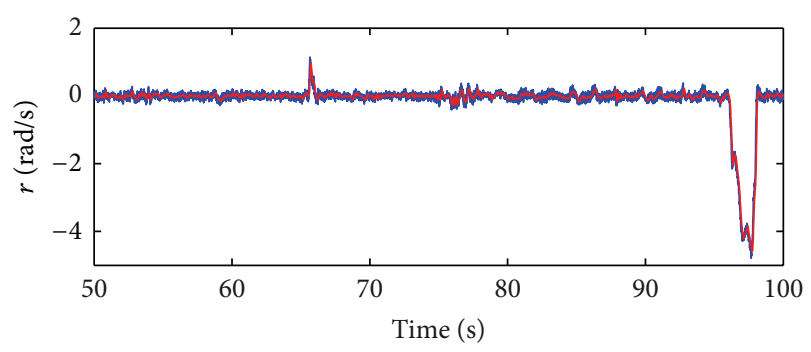

(c)

FIgURE 15: Angular rates before and after filtered.

In (14), similar to Section 3.2, a random noise $w$ with relative large variance is used to describe the dynamic of acceleration.

Based on the model in (13) and (14), a Kalman filter is designed to estimate the altitude, the performance of which will be evaluated in Section 5 .

\section{Tests and Evaluations}

In this section, a ground test and several flight tests were conducted to evaluate the performance and reliability of the overall UAV helicopter system. Ground test is mainly planned to evaluate some basic physic performance and flight tests aim at the performance of total system. The results show that the UAV helicopter we have constructed yields excellent performance.

5.1. Ground Test. The main goals of the ground test are to verify the stability of the software system and evaluate the endurance of the power system and the wireless communication range. During the ground test, the engine was governed at the normal RPM of flight, which was set to be 1650, but the main rotor blades were not installed for safety consideration.

In order to evaluate the endurance of the power system, the entire onboard avionics were powered on. The DSC 


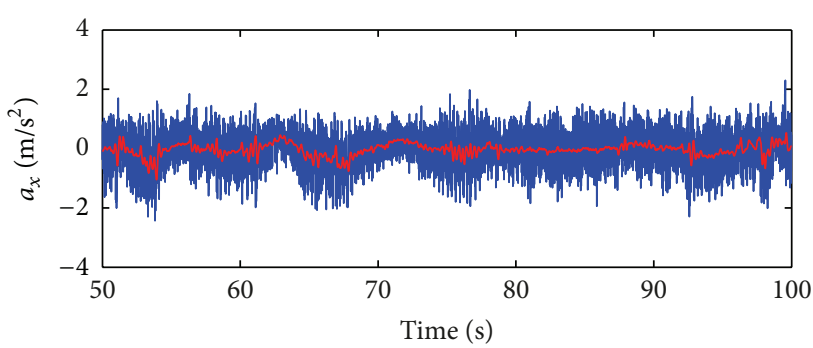

(a)

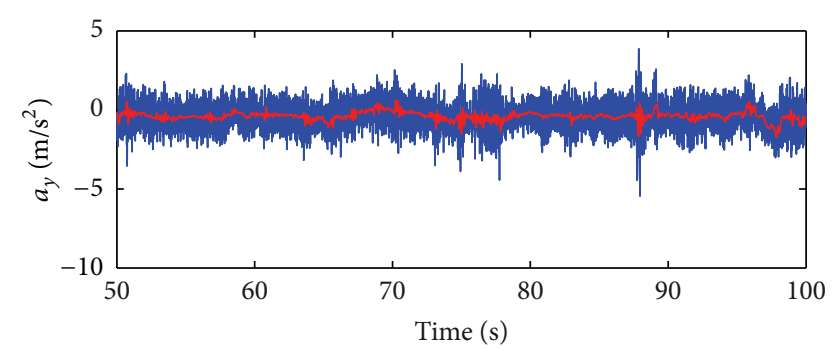

(b)

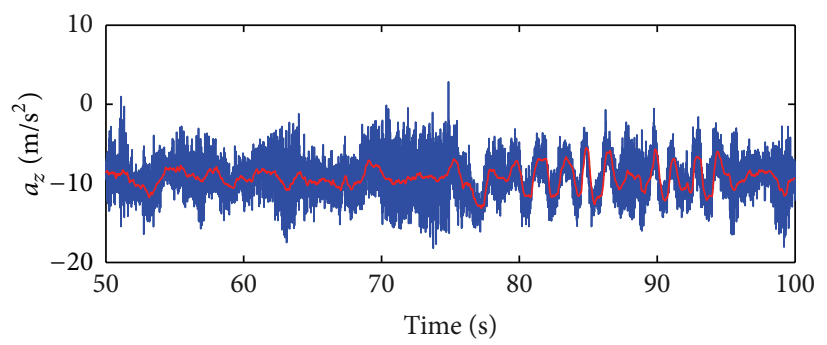

(c)

FIGURE 16: Accelerations before and after filter.

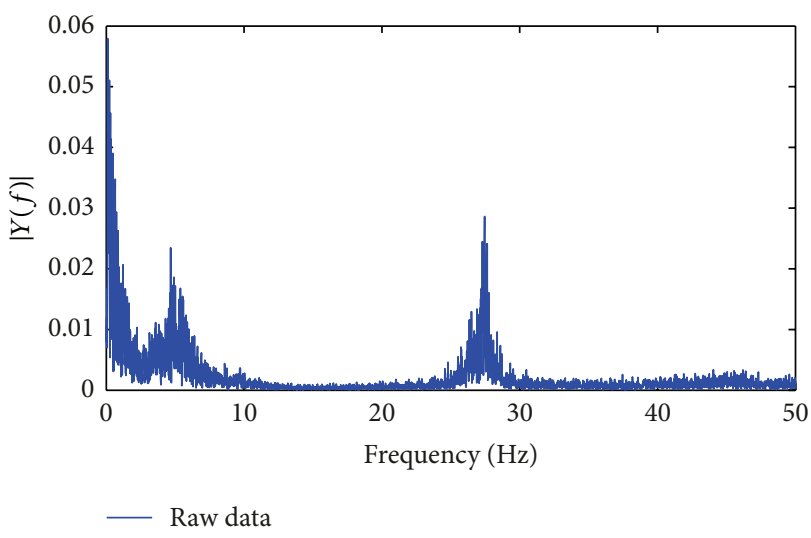

(a)

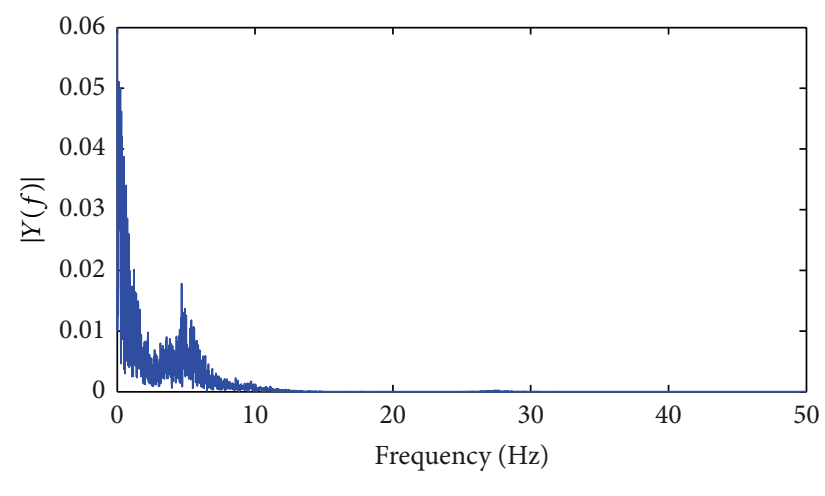

(b)

FigURE 17: FFT of raw and filtered angular rate along $x$-axis.

continuously requested data from sensors, processed and logged the collected data as well as transmitted it to ground station, which was similar to the process of real flight. The RF power of theXTend-PKG was set at $500 \mathrm{~mW}$, and the ground station was placed about $400 \mathrm{~m}$ away from the helicopter. Before the test, the 7.4 V $5600 \mathrm{mAh}$ LiPo battery had been charged fully. When the test had last about 1 hour, it still retained thirty percent capacity and the whole system worked well. The communication between onboard system and ground station was stable in the whole process. As aforementioned, the maximum flight time of the UAV helicopter is about 12 minutes. Therefore, the designed power system is enduring enough for the onboard system. We can conclude that the total system can work well even in vibration condition.
5.2. Flight Tests. After the ground test, three actual flight tests were conducted under manual control to verify the performance of the avionics and data processing and integrated navigation algorithms. The first test is designed to evaluate the reliability of the avionics in flight and the performance of MTi data processing algorithm. Several maneuvers including hovering, rolling, pitching, and yawing were performed. The UAV helicopter was first commanded to maintain in hover. Secondly, frequency-sweep signals were injected to the input channels to drive rolling, pitching, and yawing motion. A frequency-sweep signal was applied to the specific input channel. The pilot used the rest of the three channels to trim the helicopter. The second step was used to produce strong maneuverability to evaluate the feasibility of the onboard system in drastic situation. In the whole process, all flight 


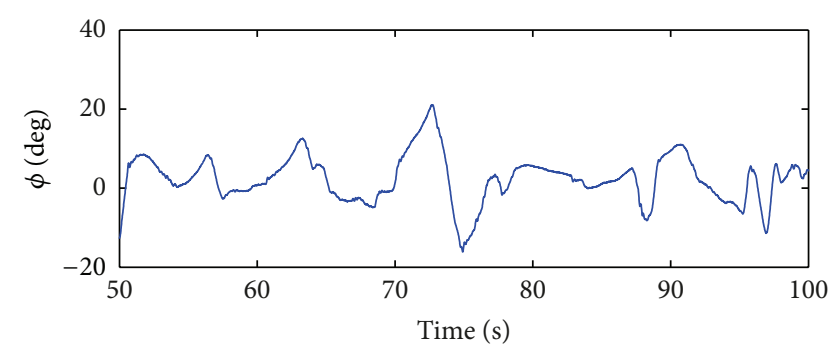

(a)

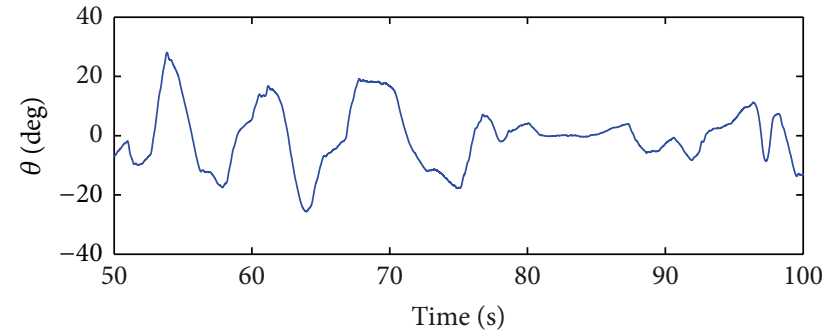

(b)

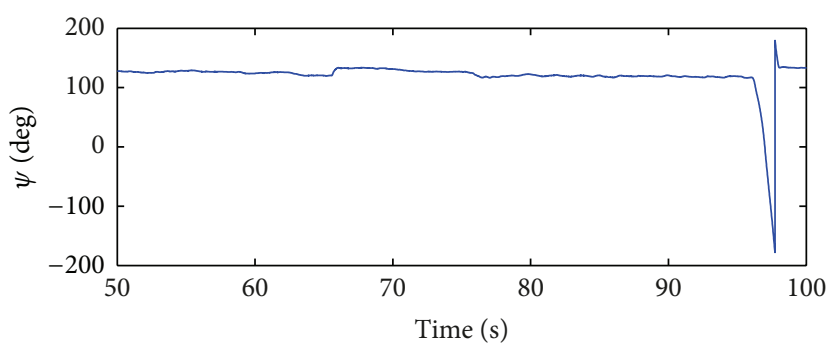

(c)

FIGURE 18: Euler angles in flight test.

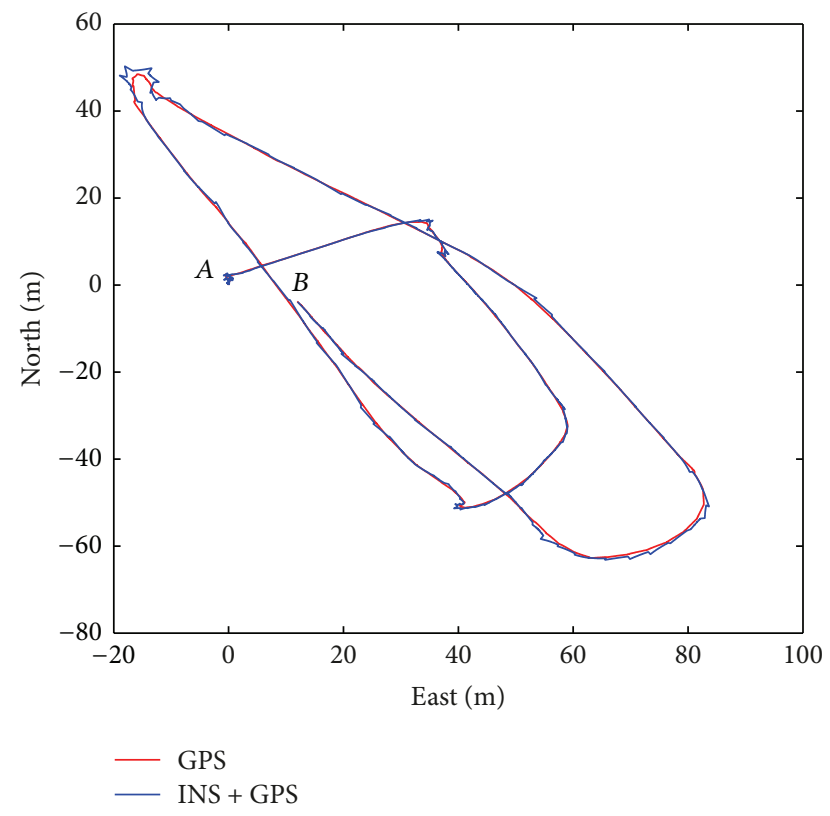

Figure 19: Trajectory of flight test.

data before and after processed and the control signals were logged into SD card. As a result, the system works well and it is suitable for real flight. Figures 14-18 show the results of the test. The test lasts for 140 seconds and the data from 50 to 100 seconds are selected for analysis. Figure 14 illustrates the duty cycle of the control inputs. The PWM signal in each subplot is used to drive the corresponding servo actuator. The angular rates and accelerations are shown in Figures 15 and 16. Blue line is used to represent the raw data from MTi directly and red line is used to represent the data filtered

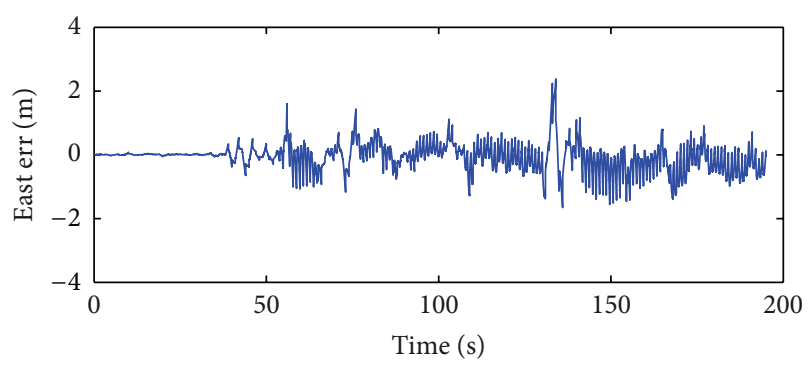

(a)

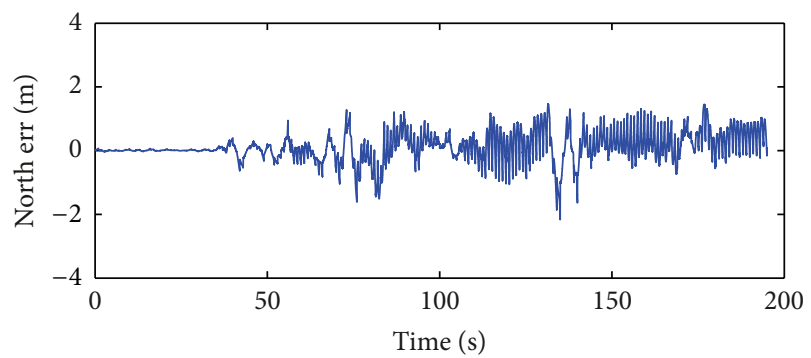

(b)

FIgURe 20: Position error.

by the two-stage cascade filters introduced in Section 3. In order to evaluate the performance of the filter, the FFT graphs of the raw and filtered angular rate along $x$-axis are plotted in Figure 17. It is clear that both the vibrations and highfrequency noises are filtered and only the useful information in selected frequency range is allowed to pass. The Euler angles are shown in Figure 18. The curves are very smooth and it seems that the angles are not affected by any noise.

The second test is mainly used to check the reliability of the integrated navigation algorithm. In this test, the UAV 


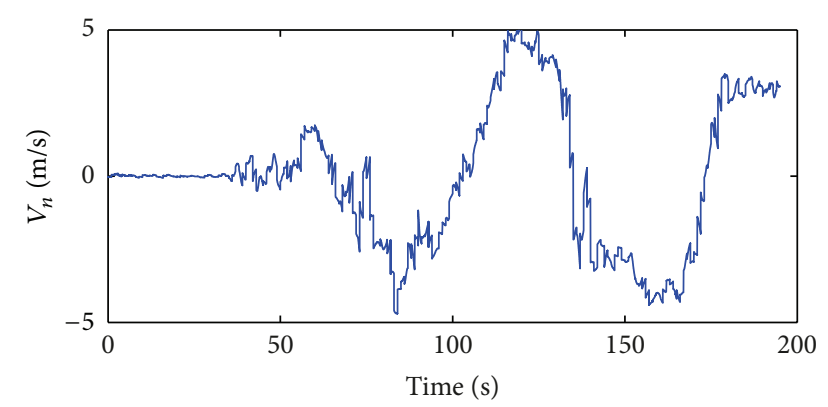

(a)

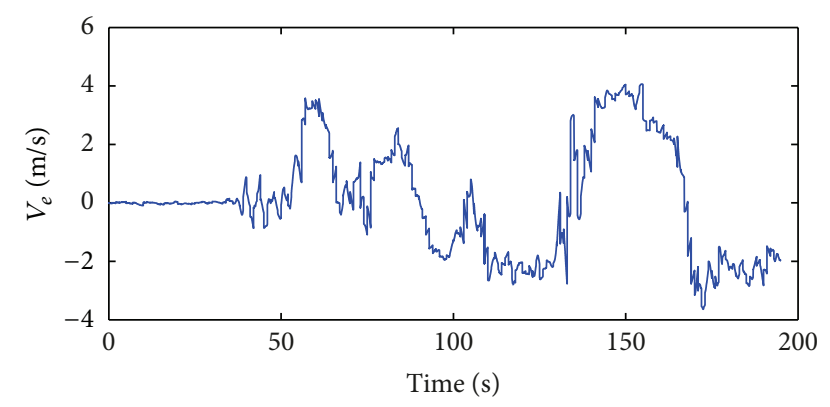

(b)

FIGURE 21: Integrated navigation velocity.

helicopter was manual controlled by a skilled pilot to track a specified trajectory. This test was conducted when the number of the GPS satellites had been more than 8 so that the position accuracy of GPS can be guaranteed. The raw data of GPS module and the output of the integrated navigation algorithm were both logged. As shown in Figure 19, the UAV helicopter was placed at point A. It took about 35 seconds to take off. After taking off, the pilot maintained it in hover carefully. About 10 seconds later, it was controlled to track the specified trajectory. We stopped to logged data and ended the test when it reached point $B$. Red line represents the raw position data from GPS module and blue line represents the integrated navigation position. The errors between the GPS position and the integrated navigation position are shown in Figure 20, which are mainly limited in $\pm 1 \mathrm{~m}$ and enough for flight control. The velocities along east and north in gauss coordinate frame are shown in Figure 21.

The purpose of the third test is to evaluate the performance of the altitude estimation. In this test, the UAV helicopter was mainly controlled to maneuver in altitude channel. The result is shown in Figure 22. It's clear that there is a bias between barometric altitude and GPS altitude and the relative altitude change is mainly corresponding to the barometric altitude. Actually, barometric altimeter is more accurate than GPS. And the estimated result is acceptable for system identification and flight control.

\section{Conclusions}

In this paper, a small-scale UAV helicopter platform has been constructed. Both the hardware and software parts of

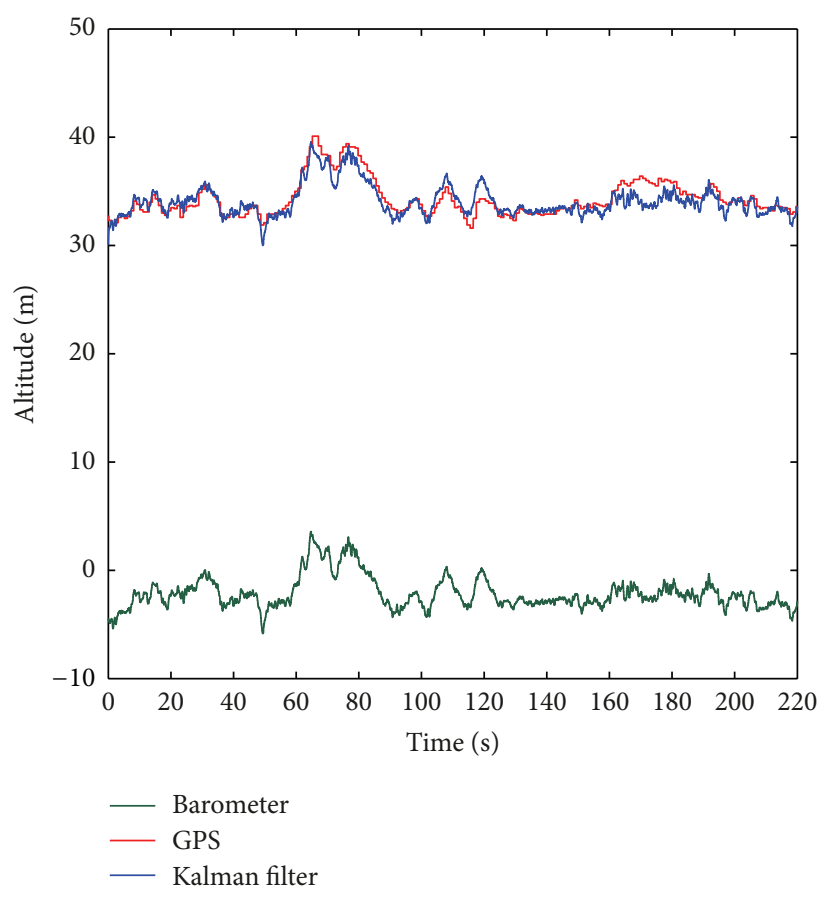

FIGURE 22: Altitude of UAV helicopter in flight.

the onboard system are designed and have been successfully implemented. The hardware part comprises bare hobby helicopter, flight control board, avionic sensors, and RF modem. All the components are selected or designed carefully with the attributes of compact and low cost. The ground station is designed to monitor the status of the UAV helicopter, which consists of a computer and a RF modem. Then, MTi data processing algorithm and integrated navigation algorithm are designed and implemented to estimate the states of helicopter. At last, several ground and flight tests are conducted to evaluate the feasibility and reliability. The results show the performance is very well and the data we can collect is sufficient for future work.

In the future, we plan to identify the mathematical model of the UAV helicopter based on the constructed platform. Furthermore, we will focus on the control law design to realize autonomous flight. In addition, a camera will be instrumented and some researches on image guidance will be carried out.

\section{References}

[1] P. Doherty, G. Granlund, K. Kuchcinski et al., "The WITAS unmanned aerial vehicle project," in Proceedings of the 14th European Conference of Articial Intelligence, August 2000.

[2] M. Bernard, K. Kondak, and G. Hommel, "Load transportation system based on autonomous small size helicopters," in Proceedings of the 23rd International Unmanned Air Vehicle Systems Conference, Bristol, UK, April 2008.

[3] A. R. Girard, A. S. Howell, and J. K. Hedrick, "Border patrol and surveillance missions using multiple unmanned air vehicles," in Proceedings of the 43rd IEEE Conference on Decision and Control (CDC '04), pp. 620-625, Atlantis, Bahamas, December 2004. 
[4] D. W. Casbeer, D. B. Kingston, R. W. Bear, T. W. McLain, and S. M. Li, "Cooperative forest fire surveillance using a team of small unmanned air vehicles," International Journal of Systems Science, vol. 37, no. 6, pp. 357-360, 2006.

[5] S. K. Kim and D. M. Tilbury, "Mathematical modeling and experimental identification of a model helicopter," AIAA, 1998.

[6] V. Gavrilets, A. Shterenberg, M. A. Dahleh, and E. Feron, "Avionics system for a small unmanned helicopter performing agressive maneuvers," in Proceedings of the 19th Digital Avionics Systems Conference, Philadelphia, Pa, USA, October 2000.

[7] K. Sprague, V. Gavrilets, D. Dugail, B. Mettler, E. Feron, and I. Martinos, "Design and applications of an avionics system for a miniature acrobatic helicopter," in Proceedings of the 20th AIAA Digital Avionics Systems Conference, pp. 3-C51, Daytona Beach, Fla, USA, October 2001.

[8] O. Amidi, T. Kanade, and R. Miller, "Miller Vision-based Autonomous helicopter research at Carnegie Mellon Robotics institute," in Proceedings of the Heli Japan '98, Gifu, Japan, April 1998.

[9] O. Amidi, An autonomous vision-guided helicopter [Ph.D. thesis], Carnegie Mellon University, 1996.

[10] B. Mettler, Modeling small-scale unmanned rotorcraft for advanced flight control design [Ph.D. thesis], Carnegie Mellon University, 2001.

[11] J. S. Dittrich and E. N. Johnson, "Multi-sensor navigation system for an autonomous helicopter," in Proceedings of the AIAA/IEEE Digital Avionics Systems Conference, p. 8, October 2002.

[12] H. B. Christophersen, W. J. Pickell, A. A. Koller, S. K. Kannan, and E. N. Johnson, "Small adaptive flight control systems for UAVs using FPGA/DSP technology," in Proceedings of the AIAA 3rd "Unmanned-Unlimited" Technical Conference, Workshop, and Exhibit, pp. 780-787, Chicago, Ill, USA, September 2004.

[13] J. S. Dittrich and E. N. Johnson, "Multi-sensor navigation system for an autonomous helicopter," in Proceedings of the Digital Avionics Systems Conference, p. 8, October 2002.

[14] G. Cai, L. Feng, B. M. Chen, and T. H. Lee, "Systematic design methodology and construction of UAV helicopters," Mechatronics, vol. 18, no. 10, pp. 545-558, 2008.

[15] G. Cai, K. Peng, B. M. Chen, and T. H. Lee, "Design and assembling of a UAV helicopter system," in Proceedings of the 5th International Conference on Control and Automation (ICCA '05), pp. 697-702, Budapest, Hungary, June 2005.

[16] G. Cai, B. M. Chen, T. H. Lee, and M. Dong, "Design and implementation of a hardware-in-the-loop simulation system for small-scale UAV helicopters," Mechatronics, vol. 19, no. 7, pp. 1057-1066, 2009.

[17] M. Dong, B. M. Chen, G. Cai, and K. Peng, "Development of a real-time onboard and ground station software system for a UAV helicopter," Journal of Aerospace Computing, Information and Communication, vol. 4, no. 8, pp. 933-955, 2007.

[18] E. Stingu and F. L. Lewis, "A hardware platform for research in Helicopter UAV control," Journal of Intelligent and Robotic Systems, vol. 54, pp. 387-406, 2009.

[19] B. Godbolt, N. I. Vitzilaios, and A. F. Lynch, "Experimental validation of a helicopter autopilot design using model-based PID control," Journal of Intelligent and Robotic Systems, vol. 70, pp. 385-399, 2012.

[20] R. D. Garcia and K. P. Valavanis, "The implementation of an autonomous helicopter testbed," Journal of Intelligent and Robotic Systems, vol. 54, pp. 423-454, 2008.
[21] Z. Taha, Y. R. Tang, and K. C. Yap, "Development of an onboard system for flight data collection of a small-scale UAV helicopter," Mechatronics, vol. 21, no. 1, pp. 132-144, 2011.

[22] P.-J. Bristeau, E. Dorveaux, D. Vissière, and N. Petit, "Hardware and software architecture for state estimation on an experimental low-cost small-scaled helicopter," Control Engineering Practice, vol. 18, no. 7, pp. 733-746, 2010.

[23] X. Shen, Research on SINS/GPS navigation for small-sized unmanned helicopter [M.S. thesis], Zhe Jiang University, 2012.

[24] Y. Xie, Z. H. Chen, and W. Chen, "Method of altitude measure system fusion for certain unmanned helicopter," Ordnance Industry Automation, vol. 29, no. 2, pp. 85-90, 2010.

[25] B. Mettler, Identification Modeling and Characteristics of Miniature Rotorcraft, Kluwer Academic Publishers, 2003. 

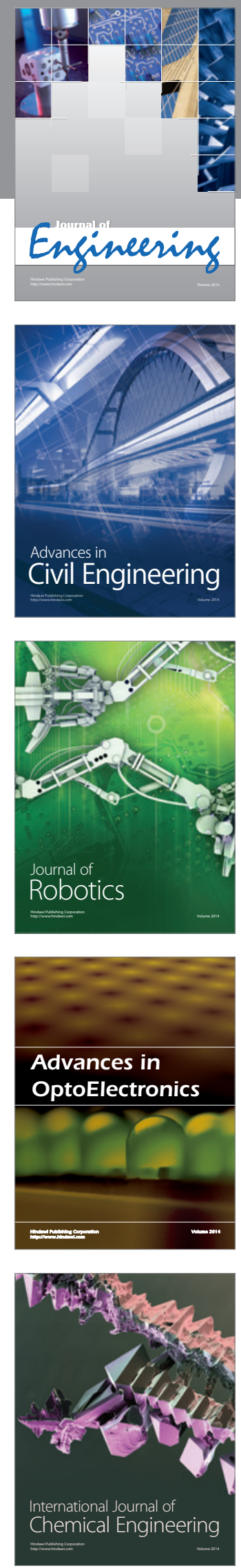

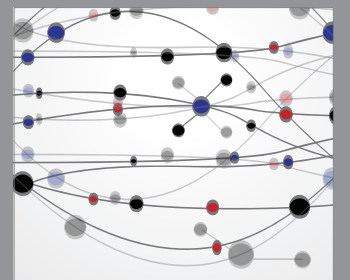

The Scientific World Journal
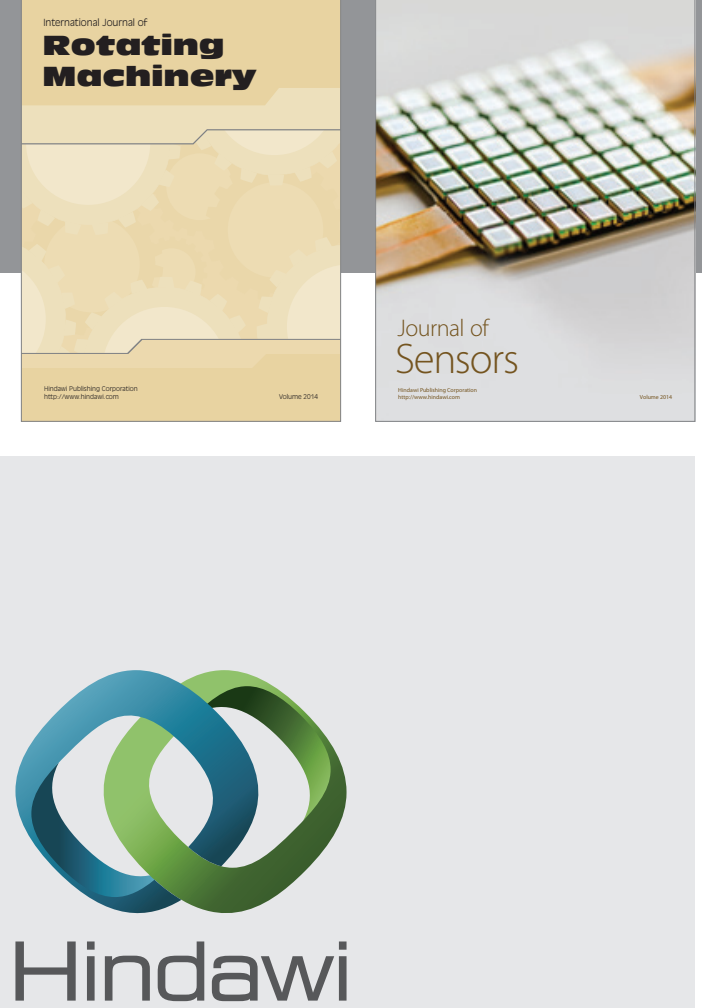

Submit your manuscripts at http://www.hindawi.com
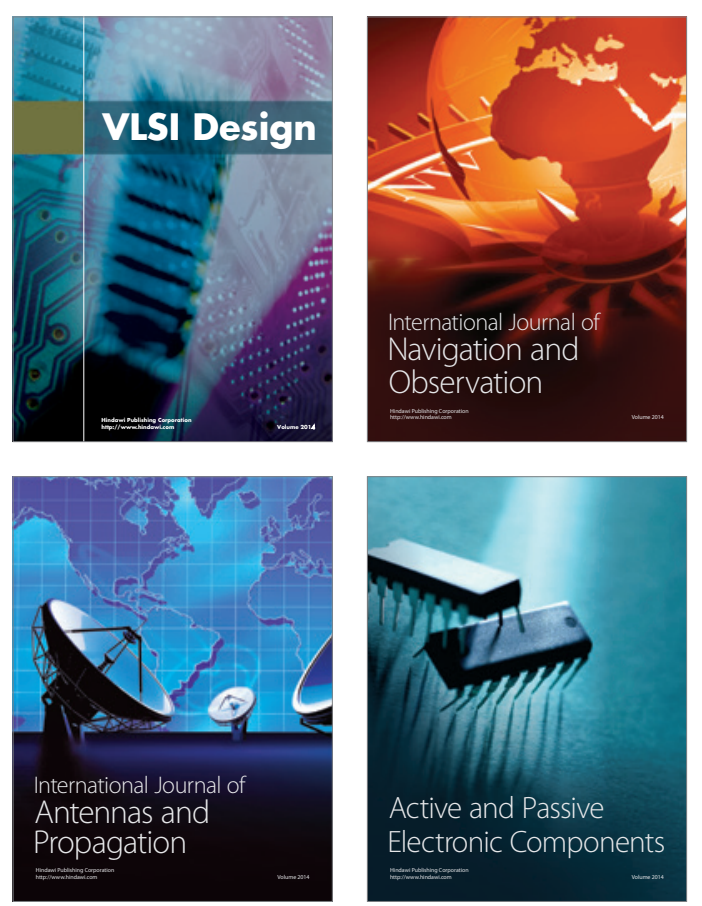
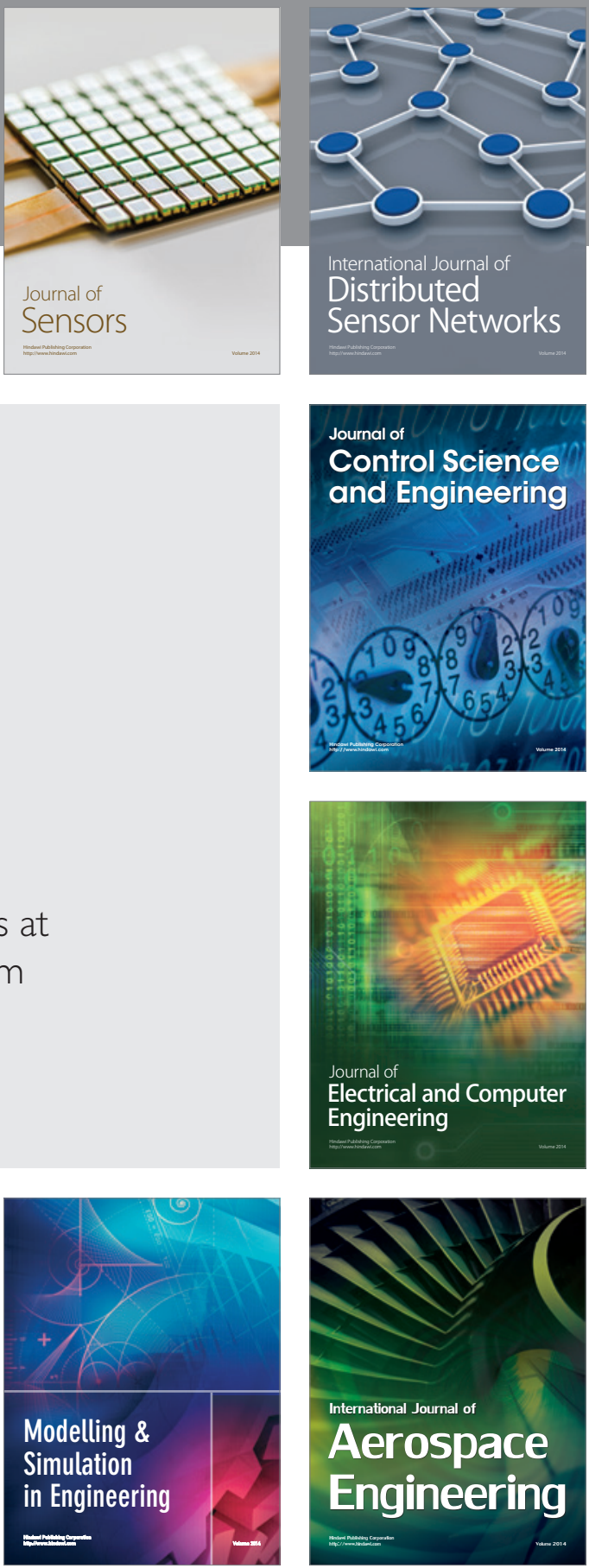

Journal of

Control Science

and Engineering
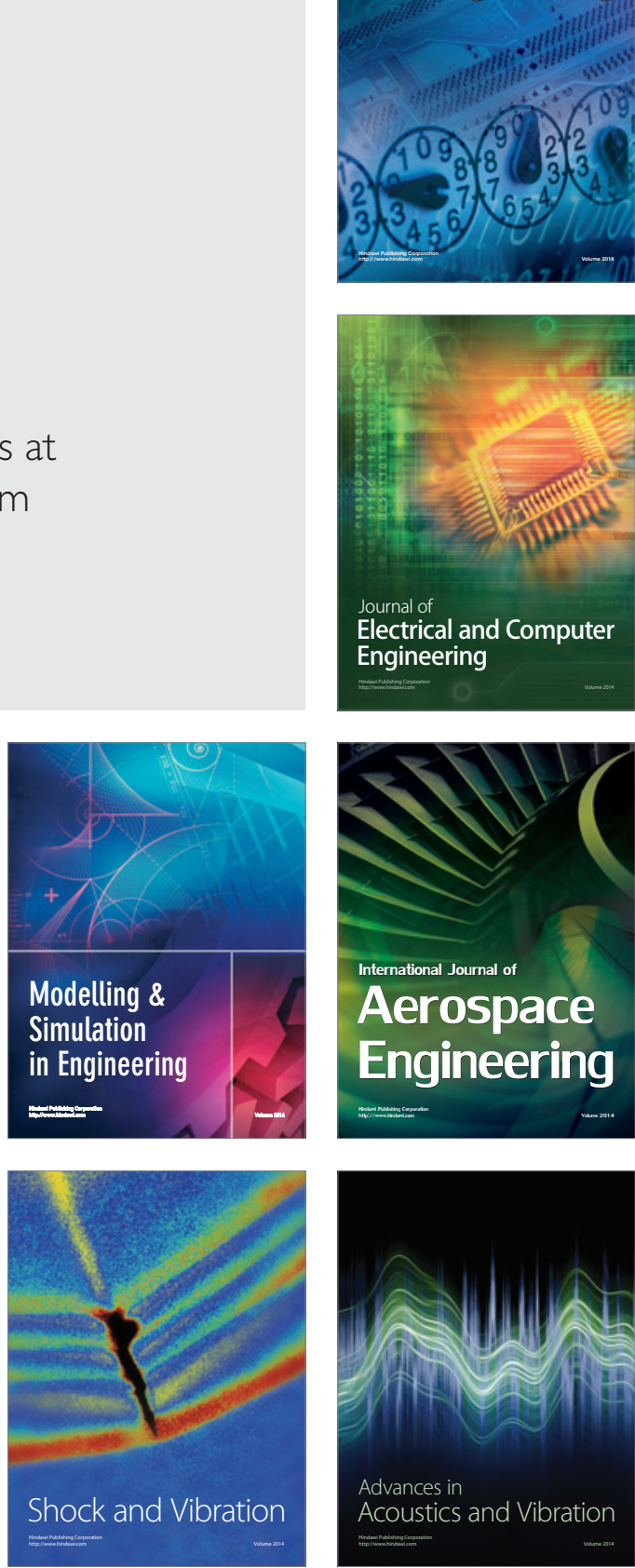